\title{
Recurrence of hepatocellular carcinoma following deceased donor liver transplantation: case series
}

\author{
Cem Simsek', Amy Kim¹, Michelle Ma', Nilay Danis', Merve Gurakar², Andrew M. Cameron ${ }^{3}$, Benjamin \\ Philosophe $^{3}$, Jacqueline Garonzik-Wang ${ }^{3}$, Shane Ottmann ${ }^{3}$, Ahmet Gurakar ${ }^{1}$, Behnam Saberi' \\ IJohns Hopkins University School of Medicine, Division of Gastroenterology and Hepatology-Transplant Hepatology, Baltimore, MD \\ 21205, USA. \\ ${ }^{2}$ Johns Hopkins University Bloomberg School of Public Health, Baltimore, MD 21205, USA. \\ ${ }^{3}$ Johns Hopkins University School of Medicine, Division of Transplant Surgery, Baltimore, MD 21205, USA.
}

Correspondence to: Dr. Ahmet Gurakar, Division of Gastroenterology and Hepatology, The Johns Hopkins University School of Medicine, 720 Rutland Avenue Ross Research Building, Suite 918 Baltimore, MD 21205, USA. E-mail: aguraka1@jhmi.edu

How to cite this article: Simsek C, Kim A, Ma M, Danis N, Gurakar M, Cameron AM, Philosophe B, Garonzik-Wang J, Ottmann S, Gurakar A, Saberi B. Recurrence of hepatocellular carcinoma following deceased donor liver transplantation: case series. Hepatoma Res 2020;6:11. http://dx.doi.org/10.20517/2394-5079.2019.51

Received: 20 Dec 2019 First Decision: 3 Feb 2020 Revised: 2 Mar 2020 Accepted: 3 Mar 2020 Published: 20 Mar 2020

Science Editor: Guang-Wen Cao Copy Editor: Jing-Wen Zhang Production Editor: Tian Zhang

\begin{abstract}
Aim: We aimed to study the clinical and pathological characteristics of liver transplant recipients with hepatocellular carcinoma recurrence.

Methods: We reviewed the data for 26 patients who had tumor recurrence after deceased donor liver transplant for hepatocellular carcinoma at the Johns Hopkins Hospital from January 2005 to December 2015.

Results: In total, $88 \%$ of recipients were males. The mean age was 59 years. On explant, poor differentiation was detected in $43 \%$, while $73 \%$ had microvascular invasion. Overall, $62 \%$ were diagnosed to be outside of Milan criteria. Out of these, $15 \%$ met the criteria for downstaging. Twenty (77\%) patients had pre-transplant alpha fetoprotein levels $\geq 20 \mathrm{ng} / \mathrm{mL}$. In 54\% of patients, the location of hepatocellular carcinoma (HCC) recurrence was extrahepatic, followed by intrahepatic in $31 \%$ and both intra- and extrahepatic in 15\%. The post-transplant tumor recurrence was diagnosed at a mean of 427 days (range 34-1502). Fifty percent of HCC recurrences were diagnosed within one year following liver transplant. Twenty (77\%) patients received treatment for their recurrent HCC: external radiation $(n=10)$, surgical resections ( $n=8$; brain 4, spine 2, bone 1, and Whipple surgery 1$)$, sorafenib $(n=7)$, locoregional therapy $(n=5)$. Overall, 24 out of 26 (92\%) recipients died within four years after the transplant.
\end{abstract}

Conclusion: HCC recurrence after liver transplant is infrequent. More than fifty percent of HCC recurrences following liver transplant are extrahepatic. Despite better recipient selection for liver transplant, the curative options are limited in recurrent cases and associated with extremely poor outcomes.

Keywords: Hepatocellular carcinoma, liver transplant, liver resection, locoregional therapy
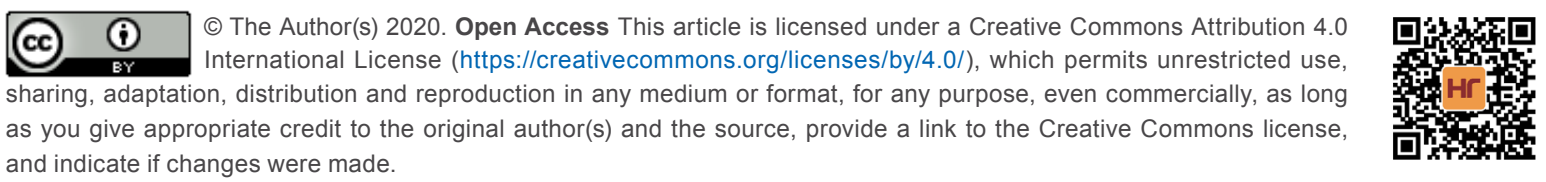
and indicate if changes were made. 


\section{INTRODUCTION}

Liver transplant (LT) has become the treatment of choice in patients with hepatocellular carcinoma (HCC) and cirrhosis who meet the Milan criteria $(\mathrm{MC})^{[1]}$. Although additional extended criteria models have been proposed, HCC recurrence following LT remains an unfortunate incident associated with poor survival ${ }^{[2,3]}$. Tumor biology and alpha fetoprotein (AFP), as well as tumor size and number, have been proposed by various groups as other potentially relevant factors of tumor recurrence ${ }^{[4-6]}$.

Overall, two thirds (2/3) of patients, who develop recurrent HCC post-LT, present with extrahepatic recurrence ${ }^{[7,8]}$. The treatment of choice in post LT HCC recurrence is determined based on the site and the extent of the recurrence ${ }^{[8]}$. However, treatments are not standardized and mostly based on expert opinion and retrospective studies ${ }^{[9]}$. Surgical treatment options have been proposed with promising outcomes in selected patients ${ }^{[10,11]}$. Locoregional therapy options, transarterial chemoembolization, radiofrequency ablation, and stereotactic radiation are considered in selected cases ${ }^{[9]}$.

In a recent report, we published our experience in LT recipients with HCC at the Johns Hopkins University Comprehensive Liver Transplant Center ${ }^{[12]}$. As a follow-up study, we aimed to study the clinicopathological features and outcomes of 26 cases with HCC recurrence following LT. In addition, we evaluated the details on the outcomes and the application of different treatment modalities in this group.

\section{METHODS}

The study was approved by the institutional review board at the Johns Hopkins Hospital. HCC-related deceased donor LT recipients between January 2005 and December 2015 were evaluated. In total, 26 patients with post-LT HCC recurrence were identified among 165 recipients who were included in the study. All recipients were listed following a standard work up and discussion at the weekly selection meeting. They were within Milan criteria or downstaged into Milan criteria. The transplant was performed by piggyback technique. Postoperative HCC surveillance consisted of contrasted cross-sectional imaging with computerized tomography or magnetic resonance imaging with AFP every three months for the first year and every six months for the second and third years. There was no set therapeutic protocol for recurrence; treatment options were discussed in a multidisciplinary fashion. The Pre-LT AFP was obtained within the past three months prior to deceased donor liver transplantation (DDLT) and immediate post-LT AFP was obtained within three months post DDLT.

Data on clinical, radiologic, pathology, HCC recurrence, and survival were collected from the records, reviewed, and analyzed. Explant pathologies were reviewed retrospectively, and the following tumor parameters were collected: size, number of lesions, microvascular invasion status, and differentiation. It was determined whether patients met the Milan or University of California San Francisco (UCSF) criteria based on the number and size of HCC lesions on explant pathology. The data collected for categorical variables were reported as percentages. Data for continuous variables were reported by the mean and standard deviation. Patient survivals were analyzed using Kaplan-Meier statistics. STATA V.13 (StataCorp college station, TX) was used to perform the statistical analyses.

\section{RESULTS}

\section{Patient characteristics}

Among the deceased donor LT recipients, HCC was the primary indication for transplantation varying from $21 \%$ to $53 \%$ of patients [Figure 1] according to the year. Clinical information on the 26 LT recipients with recurrent HCC is summarized in Table 1. Patients were predominantly male (88.5\%) with a mean age of 59 years (range 47-72 years). The majority of recipients were white $(n=17,65.4 \%)$, followed by African American $(n=7,27.0 \%)$ and Asian $(n=2,7.6 \%)$ ethnicities. Primary etiology of liver disease was chronic 


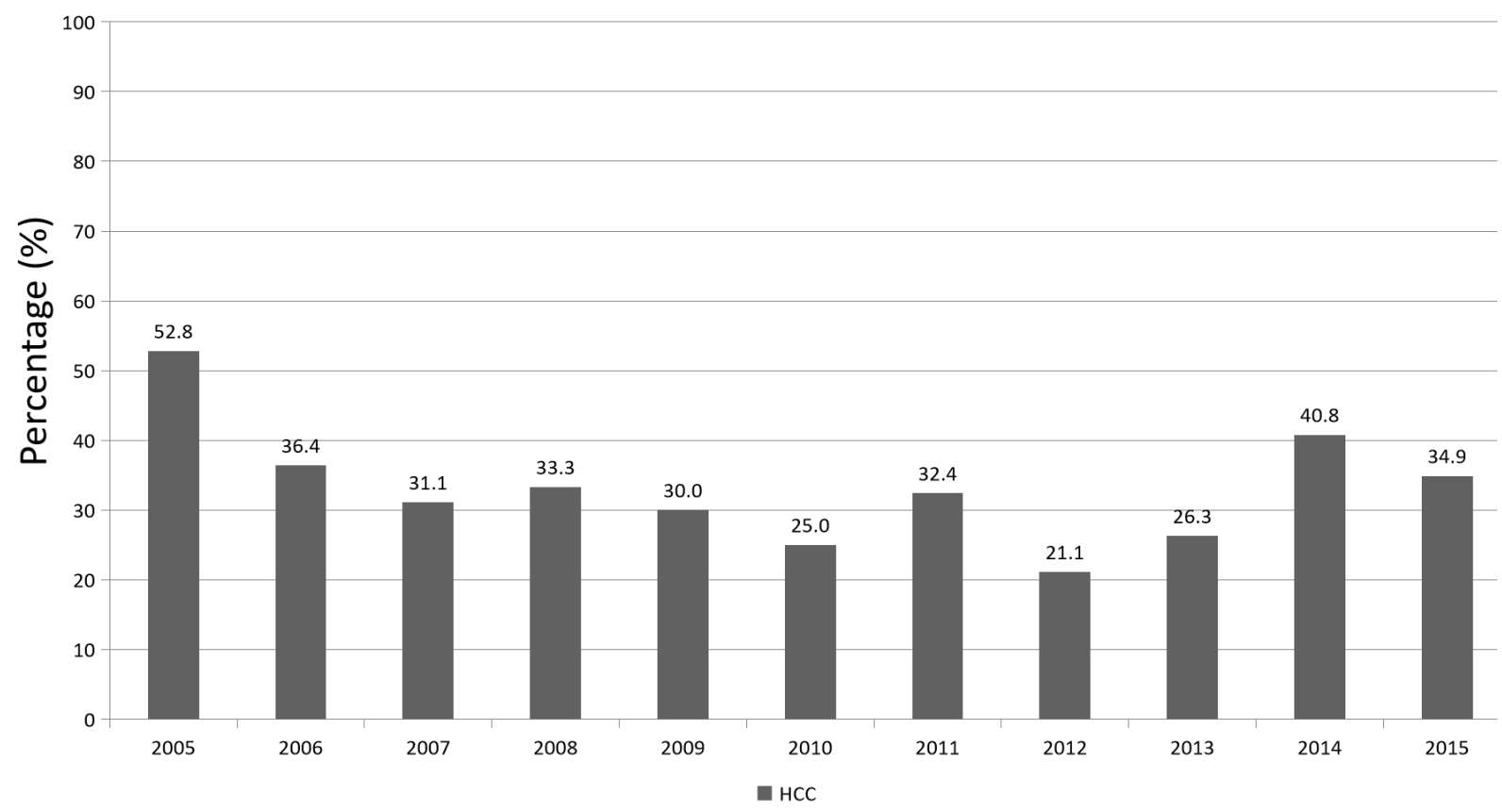

Figure 1. Overall, rate of deceased donor liver transplant for hepatocellular carcinoma indication at the Johns Hopkins Hospital from 2005 to 2015. HCC: hepatocellular carcinoma

hepatitis C (positive hepatitis C antibody and/or hepatitis C RNA) in 13 patients (50\%) and hepatitis C and alcoholic liver disease in $6(23 \%)$ patients. Chronic hepatitis B (positive hepatitis B surface antigen and/or hepatitis B DNA) was seen in three patients (11.5\%), followed by alcoholic liver disease $(n=2,7.7 \%)$, and non-alcoholic fatty liver disease $(n=1,3.9 \%)$.

\section{Laboratory results}

The average model for end-stage liver disease (MELD) score was 13, ranging from 6 to 35. Mean AFP was $27.6 \mathrm{ng} / \mathrm{mL}$ for pre-LT vs. $23.6 \mathrm{ng} / \mathrm{mL}$ for post-LT time periods [Tables 1 and 2]. Four patients had pre-LT AFP levels of $>1000 \mathrm{ng} / \mathrm{mL}$. The other available laboratory results are summarized in Table 1.

\section{Immunosuppression}

Overall, nine (34.6\%) patients were treated with mammalian target of rapamycin (mTOR) treatment with sirolimus in eight and everolimus in one patient. Seventeen patients received Tacrolimus-based therapy.

\section{Explant-pathology findings}

In the explant pathologies of LT recipients, 9 (34.6\%) patients had only one lesion and $11(42.4 \%)$ had 4 or more lesions. The average for the largest lesion size was $4.3 \mathrm{~cm}$. In total, 12 patients (46.1\%) had multilobar tumors and 13 (50\%) had tumors that were located in the right lobe. Overall, 10 patients (38.4\%) were within MC criteria and 11 patients (42.3\%) were within UCSF criteria. Four patients (15.4\%) were downstaged to MC with locoregional treatment. Seventeen (65.4\%) patients underwent locoregional therapy before transplant. None of the tumors were well-differentiated. Overall, 14 (53.8\%) patients had moderately differentiated HCC. Eleven (42.3\%) patients had HCC with poor differentiation. Microvascular invasion was detected in 19 of the 26 cases $(73.1 \%)$ while one patient had bile duct invasion only.

\section{Recurrence and survival}

The overall rate of HCC recurrence following LT in our series was 15\%. The rate of HCC recurrence has improved over the years with a recurrence rate of $10 \%$ in 2015 [Figure 2]. Mean time for diagnosis of 
Table 1. Characteristics of the study population

\begin{tabular}{|c|c|}
\hline Variable & $n=26$ \\
\hline \multicolumn{2}{|l|}{ Clinical features } \\
\hline Male sex, $n(\%)$ & $23(88.5 \%)$ \\
\hline Age (years) & $58.9(6.8)$ \\
\hline \multicolumn{2}{|l|}{ Ethnicity, $n(\%)$} \\
\hline White & $17(65.4 \%)$ \\
\hline African American & $7(27.0 \%)$ \\
\hline Asian & $2(7.6 \%)$ \\
\hline \multicolumn{2}{|l|}{ Etiology } \\
\hline $\mathrm{HCV}$ & $13(50 \%)$ \\
\hline HBV & $3(11.5 \%)$ \\
\hline ALD & $2(7.7 \%)$ \\
\hline NAFLD & $1(3.9 \%)$ \\
\hline HCV/ALD & $6(23 \%)$ \\
\hline Other & $1(3.9 \%)$ \\
\hline \multicolumn{2}{|l|}{ Explant pathology } \\
\hline \multicolumn{2}{|l|}{ Number of lesions, $n(\%)$} \\
\hline 1 & $9(34.6 \%)$ \\
\hline 2 & $3(11.5 \%)$ \\
\hline 3 & $3(11.5 \%)$ \\
\hline$>4$ & $11(42.4 \%)$ \\
\hline Largest lesion (cm) & $4.3(3.8)$ \\
\hline \multicolumn{2}{|l|}{ Tumor location, $n(\%)$} \\
\hline Right lobe & $13(50 \%)$ \\
\hline Left lobe & $1(3.9 \%)$ \\
\hline Multi-lobar & $12(46.1 \%)$ \\
\hline \multicolumn{2}{|l|}{ Tumor differentiation, $n$ (\%) } \\
\hline Well & $0(0 \%)$ \\
\hline Moderate & $14(53.8 \%)$ \\
\hline Poor & $11(42.3 \%)$ \\
\hline Unknown & $1(3.9 \%)$ \\
\hline \multicolumn{2}{|l|}{ Microvascular invasion, $n(\%)$} \\
\hline Yes & $19(73.1 \%)$ \\
\hline No & $6(23 \%)$ \\
\hline \multirow{2}{*}{\multicolumn{2}{|c|}{ Total number of loco-regional therapies, $n(\%)$}} \\
\hline & \\
\hline 0 & $9(34.6 \%)$ \\
\hline 1 & $9(34.6 \%)$ \\
\hline 2 & $5(19.2 \%)$ \\
\hline$>2$ & $3(11.6 \%)$ \\
\hline \multicolumn{2}{|l|}{ Patients with viable tumor, $n(\%)$} \\
\hline Yes & $25(96.2 \%)$ \\
\hline No & $1(3.8 \%)$ \\
\hline \multicolumn{2}{|l|}{ Within Milan, $n(\%)$} \\
\hline Yes & $10(38.4 \%)$ \\
\hline No & $16(61.6 \%)$ \\
\hline Downstaged to Milan, $n$ (\%) & $4(15.4 \%)$ \\
\hline \multicolumn{2}{|l|}{ Within UCSF, $n(\%)$} \\
\hline Yes & $11(42.3 \%)$ \\
\hline No & $15(57.7 \%)$ \\
\hline Downstaged to UCSF, $n(\%)$ & $3(11.5 \%)$ \\
\hline \multicolumn{2}{|l|}{ Laboratory } \\
\hline Pre-LT AFP (ng/mL) & $27,578(133,183)$ \\
\hline Post-LT AFP (ng/mL) & $23,586(81,707)$ \\
\hline MELD & $13(7)$ \\
\hline WBC $\left(10^{9} / L\right)$ & $6(2.2)$ \\
\hline $\mathrm{Hgb}(\mathrm{g} / \mathrm{dL})$ & $12.9(2.7)$ \\
\hline$M C V(f L)$ & $91(6)$ \\
\hline $\operatorname{PLT}\left(10^{3} / \mu \mathrm{L}\right)$ & $116(67)$ \\
\hline $\mathrm{BUN}(\mathrm{mg} / \mathrm{dL})$ & $15(6)$ \\
\hline Creatinine (mg/dL) & $1.1(0.6)$ \\
\hline $\mathrm{TP}(\mathrm{g} / \mathrm{dL})$ & $7.2(0.8)$ \\
\hline Alb (g/dL) & $3.6(0.7)$ \\
\hline $\operatorname{ALP}(U / L)$ & $141(58)$ \\
\hline AST (U/L) & 109 (167) \\
\hline $\operatorname{ALT}(U / L)$ & $71(122)$ \\
\hline T.Bili (mg/dL) & $2.2(2.4)$ \\
\hline $\mathrm{PT}(\mathrm{sec})$ & $14(4.1)$ \\
\hline INR & $1.3(0.4)$ \\
\hline
\end{tabular}

Clinical and pathological characteristics of the 26 recipients with hepatocellular carcinoma recurrence following liver transplant. Quantitative data are expressed as mean and categorical variables are reported as percentages. AFP: alpha fetoprotein; ALD: alcoholic liver disease; Alb: albumin; ALP: alkaline phosphatase; AST: aspartate aminotransferase; ALT: alanine aminotransferase; BUN: blood urea nitrogen; HBV: hepatitis B virus; HCV: hepatitis C virus; Hgb: hemoglobin; INR: international normalized ratio; LT: liver transplant; MCV: mean corpuscular volume; MELD: model for end-stage liver disease; NAFLD: non-alcoholic fatty liver disease; PLT: platelet count; PT: prothrombin time; TP: total protein; T.Bili: total bilirubin; UCSF: University of California San Francisco; WBC: white blood cell count 
Table 2. Alpha fetoprotein levels pre and post-liver transplant

\begin{tabular}{llll}
\hline Patient & Pre-LT AFP & Initial post-LT AFP & AFP at recurrence \\
\hline 1 & 9 & 7 & 2019 \\
2 & 28,139 & 365,210 & NA \\
3 & 135 & 4.1 & 15 \\
4 & 3.6 & 0.6 & 1.7 \\
5 & 27 & 3864 & NA \\
6 & 488 & 57 & 26 \\
7 & 22 & 2 & 1416 \\
8 & 23 & 12 & 7 \\
9 & 162 & 6.4 & 3342 \\
10 & 169 & 682 & 389 \\
11 & 34 & 3 & 12 \\
12 & 48 & 4 & 157 \\
13 & 323 & 76 & NA \\
14 & 7 & 21 & 51 \\
15 & 23 & 10 & NA \\
16 & 4659 & 25,154 & 5.4 \\
17 & 304 & 35 & 210 \\
18 & 3.3 & 4.3 & 47,304 \\
19 & 1707 & 100 & 3.7 \\
20 & 34 & 2 & 120,848 \\
21 & 680,000 & 217,576 & 17 \\
22 & 22 & 2 & NA \\
23 & 4 & 2 & 40.9 \\
24 & 207 & 317 & 3677 \\
25 & 486 & 104 & 4 \\
26 & 5.2 & 5.5 & \\
\hline
\end{tabular}

Alpha fetoprotein levels $(\mathrm{ng} / \mathrm{mL}$ ) pre- and post-liver transplant in 26 liver transplant recipients with hepatocellular carcinoma recurrence. AFP: alpha fetoprotein; LT: liver transplant; NA: data not available

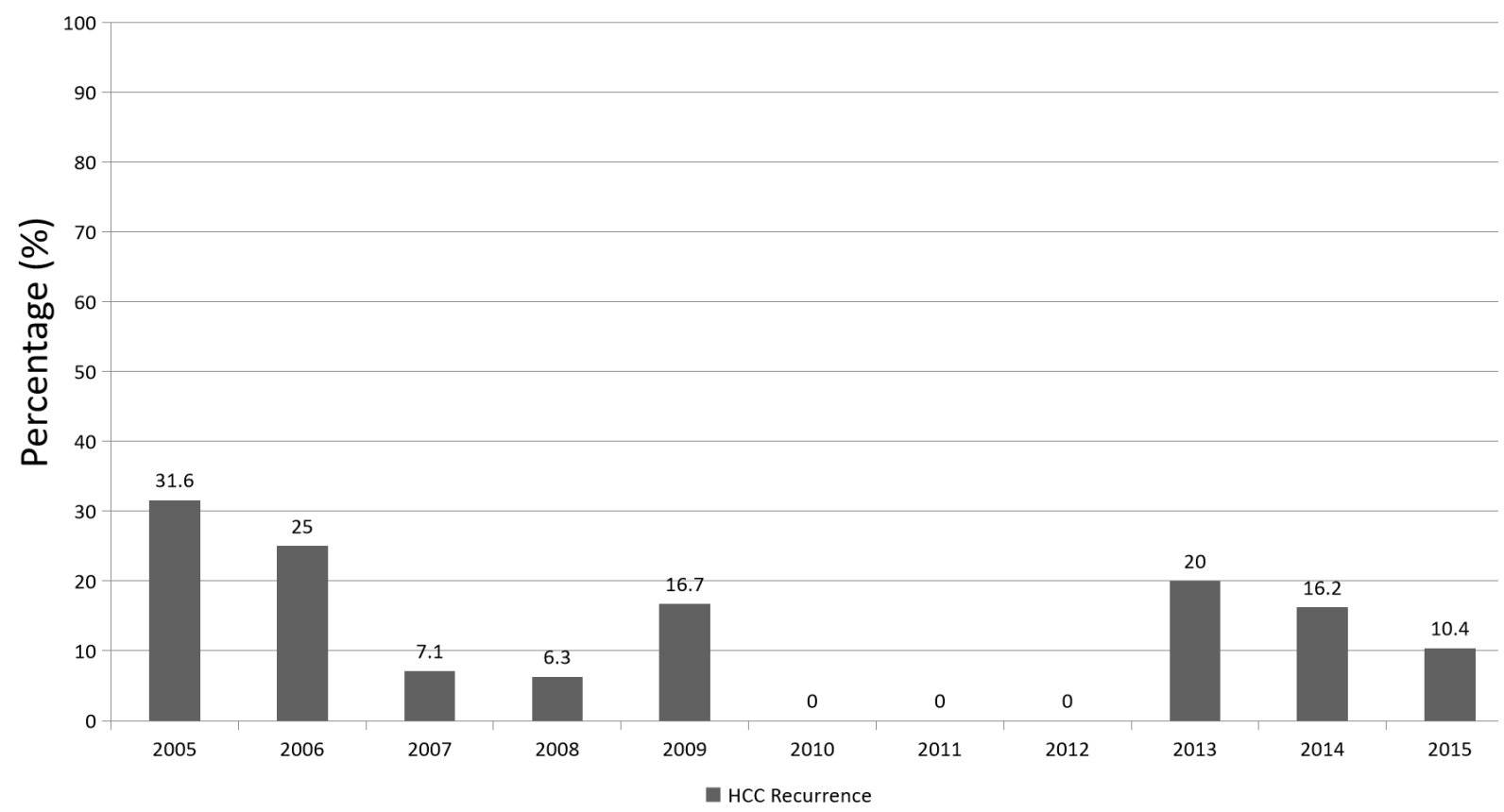

Figure 2. Overall, per year rate of hepatocellular carcinoma recurrence in deceased donor liver transplant recipients at the Johns Hopkins Hospital from 2005 to 2015. HCC: hepatocellular carcinoma 
HCC recurrence after LT was 427 days, ranging from 34 to 1502 days. The site of HCC recurrence was intrahepatic in $8(31 \%)$, extrahepatic in $14(54 \%)$, and both intra- and extrahepatic in $4(15 \%)$ patients. Overall, $31 \%$ of recipients had intrahepatic HCC recurrence following LT when compared to $69 \%$ with extrahepatic recurrence. Twenty-two percent of the patients who had extrahepatic involvement had concomitant liver involvement. The most common sites of extrahepatic involvement were the lungs (44.4\%) and bones (44.4\%) (spine, rib, pelvis, and humerus), followed by mediastinum (27.8\%), brain (22.2\%), portal lymph nodes $(11.1 \%)$, gastro-hepatic ligament (5.6\%), adrenal gland (5.6\%), pleura (5.6\%), and peritoneum $(5.6 \%)$.

A range of different treatment modalities was used for recurrences [Table 3]. Six (21.4\%) of the 26 patients were managed with supportive care. The remaining 20 cases received various treatment modalities including locoregional therapy (transarterial chemoembolization in 3, Y 90 in 1, and radiofrequency ablation in 1), external radiation in 10, and surgical resections in 8 (brain 4 , spine 2, bone 1 , and Whipple surgery in 1). Nine (32\%) patients received combination therapies of the above-mentioned modalities. Seven patients $(27 \%)$ received sorafenib. An additional two patients received chemotherapy regimens other than sorafenib [Table 3]. Recurrence-free survival and overall survival are shown in Figure 3. Patients who developed HCC recurrence following LT had an extremely poor overall survival (7.7\%). In total, 19\% of patients died within one year following LT. Overall, 24 out of 26 (92.3\%) patients died throughout the fouryear follow-up period. Timing of death relevant to the time of LT is shown in Table 3.

\section{DISCUSSION}

In this series, we report a rate of 15\% HCC recurrence following deceased donor LT at our transplant program. This rate is consistent with the literature report of $15 \%-20 \%$ post-LT HCC recurrence ${ }^{[13]}$. It is well known that the patients who are outside of MC prior to LT have higher rates of tumor recurrence following LT, compared to those within the $\mathrm{MC}^{[1]}$. Although all of the patients within our series were thought to be within MC radiographically prior to LT, according to radiology findings, only $34 \%$ were within the criteria by reviewing the explant. When including an additional four (15\%) patients who were downstaged, in total $49 \%$ were within MC based on pathology. This discrepancy between radiology and pathology has been previously described by other groups in the literature ${ }^{[13]}$.

Our sites of recurrence findings are very similar to the recent reports ${ }^{[8]}$. In a systematic review of post-LT HCC recurrence, extrahepatic site was the most common site of recurrence in $67 \%$ of cases, compared to intrahepatic in $33 \%^{[8]}$. The extrahepatic sites of involvement included: bone, pulmonary, adrenal, lymph nodes, and brain ${ }^{[8]}$.

Within our series, $54 \%$ of the HCC recurrences were diagnosed within 1 year post-OLT, while $81 \%$ and $96 \%$ of recurrences occurred within 2 and 3 years following OLT, respectively. The average time to HCC recurrence within our series was 427 days (range 34-1502 days). It is shown by others that early versus late recurrence is a predictor of post-LT survival ${ }^{[14]}$. The patients with early HCC recurrence, defined as recurrence within 24 months post-LT, have a worse prognosis ${ }^{[14]}$. There are a few potential theories for early HCC recurrence post-LT: (1) biologically rapid growing, aggressive tumors; (2) lack of high-quality pre-LT imaging or overlooking intra- or extrahepatic imaging ${ }^{[8]}$; (3) extrahepatic microscopic viable HCC cells that could not be detected by conventional imaging prior to LT; and (4) presence of circulating tumor cells that seed to other sites. The mechanism by which the late recurrence occurs is unclear ${ }^{[15]}$. Presence of pre-LT HCCs that are biologically slow growing, or development of de novo HCC recurrence in the liver allograft could be the cause. Within our series, we did not have any cases who had HCC recurrence that occurred or were diagnosed beyond five years following LT.

The selection of an ideal treatment for post LT HCC recurrence is a matter of debate, and the evidence is mainly based on expert opinion and non-randomized cohort studies ${ }^{[9]}$. The treatment modality will vary 


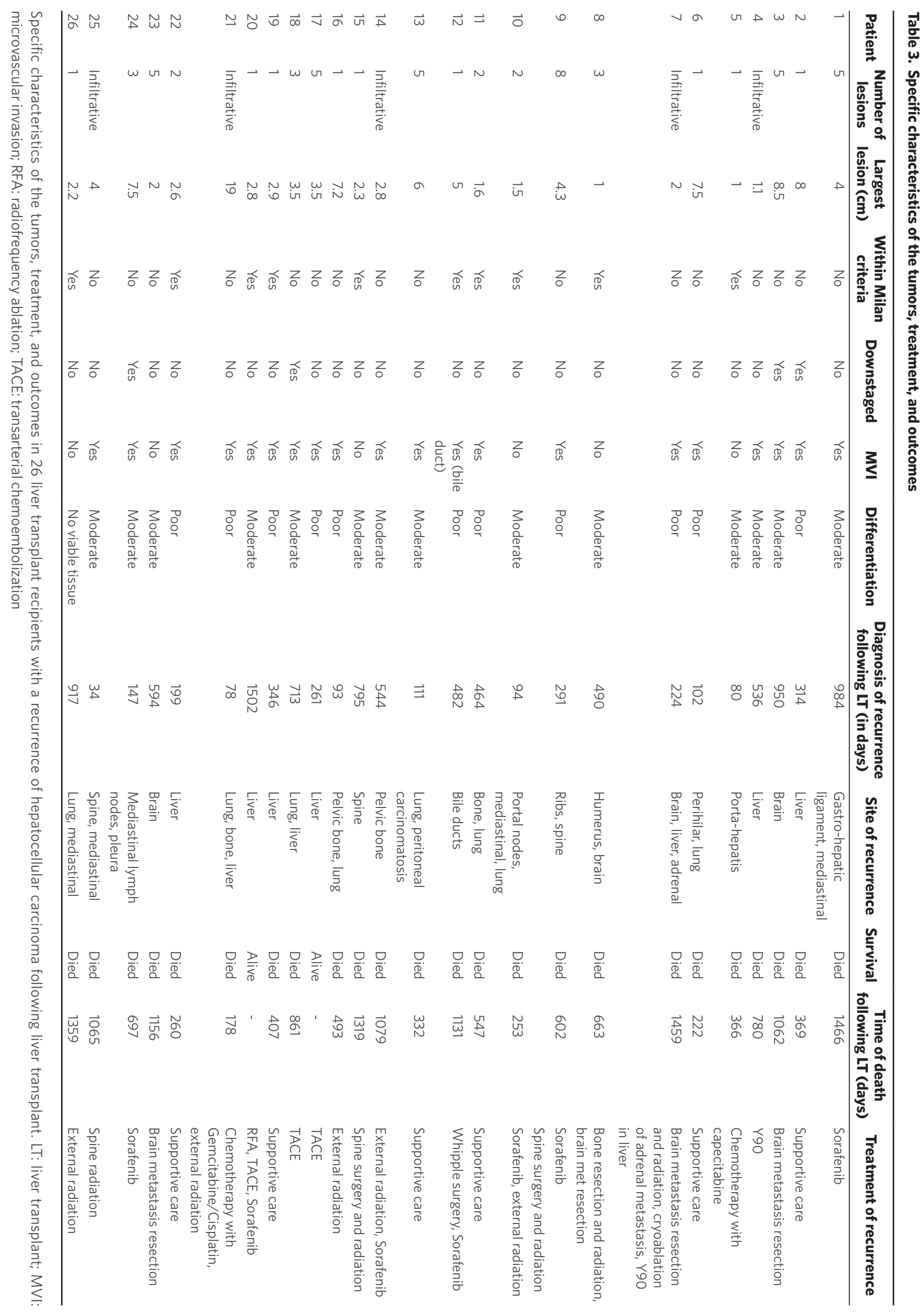



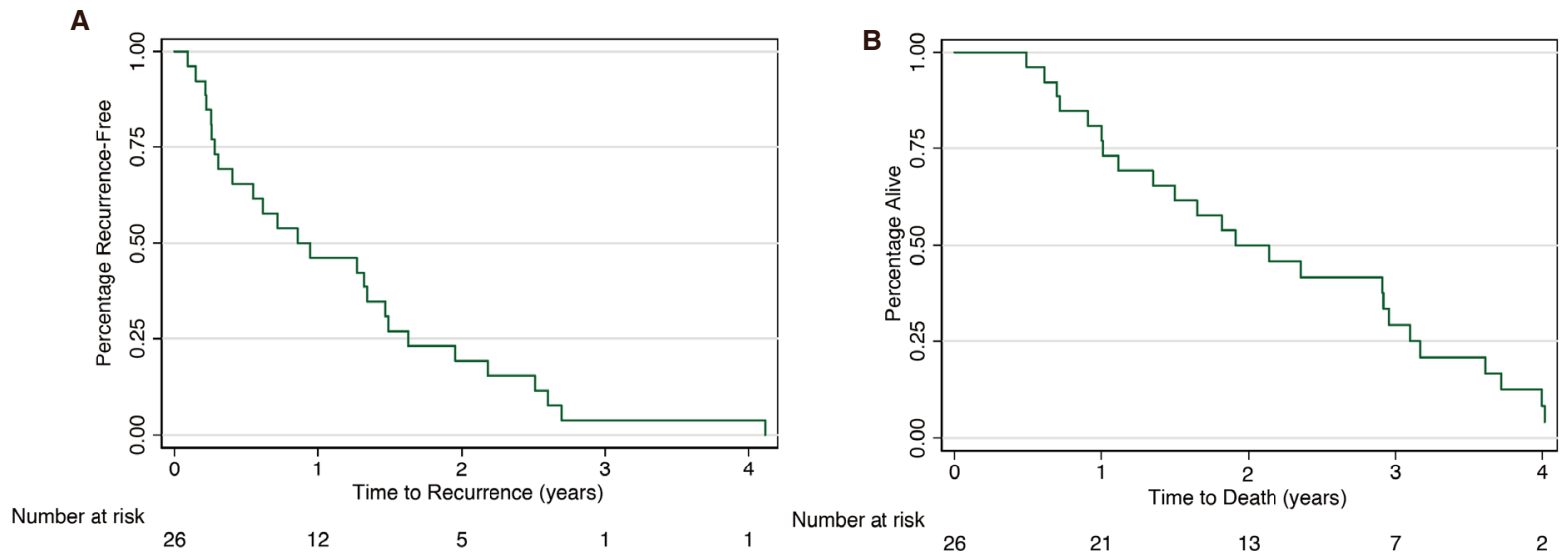

Figure 3. Survival analysis for 26 liver transplant recipients with hepatocellular carcinoma recurrence: A: Kaplan-Meier curve for recurrence-free survival; B: Kaplan-Meier curve for overall survival

based on the type of recurrence (intrahepatic versus extrahepatic), organ of involvement, and extent of involvement. This includes a wide range of surgical (intra- or extrahepatic resection and re-transplantation) and non-surgical treatments (locoregional therapies, sorafenib, other systemic chemotherapy, mTOR inhibitors, and best supportive care $)^{[16]}$.

Surgical options including extrahepatic resection, liver graft resection, and liver re- transplant have also been considered for patients presenting with HCC recurrence. In 2004, the Mount Sinai group reported resection of the liver allograft in five out of 18 recipients with HCC recurrence ${ }^{[11]}$. The authors concluded that, in selected cases with recurrent intrahepatic-HCC, liver resection improved survival ${ }^{[11]}$ Similarly, Kornberg et al. ${ }^{[10]}$ reported that HCC recurrence should be treated surgically in eligible patients with good long-term outcomes. In multivariate analysis of post-LT HCC recurrence, late tumor recurrence ( $>24$ months) and surgical resection were the two independent predictors of survival ${ }^{[10]}$. A systematic review in 2015 reported that the surgical approach to localized intra- or extra-hepatic recurrences are uneventful and not associated with higher mortality ${ }^{[8]}$. Retransplantation for recurrent HCC is not a practical option ${ }^{[17]}$ due to the higher risk of recurrence with a limited organ availability.

Sorafenib, a multikinase inhibitor, has been approved as first-line treatment for the management of advanced-stage HCC following two clinical trials in 2008 and $2009^{[18,19]}$. In a multicenter phase 2, blinded placebo-controlled, clinical trial, the efficacy of sorafenib for preventing HCC recurrence post-LT in highrisk recipients is being actively investigated [ClinicalTrials.gov identifier (NCT number): NCTo1624285]. There are currently no systemic therapies that have been shown to improve survival in HCC recurrence post-LT. Recently, other tyrosine kinase inhibitors were approved as first- or second-line treatment in HCC in the non-transplant setting ${ }^{[20]}$. The role of these agents as adjuvant therapy or post-LT HCC recurrence is unclear and deserves further investigation in the near future. Nivolumab, an anti-PD 1 inhibitor, was recently approved for the treatment of HCC, as second line, in the non-transplant setting, with the objective response rate of $20 \%{ }^{[21]}$. The role of immunotherapy among post-LT recipients with HCC has not been yet established. It is possible that the immunotherapy will affect the liver allograft leading to acute cellular rejection $^{[22]}$.

Mammalian target of rapamycin (mTOR), a serine/threonine protein kinase, has been shown to be upregulated in $40 \%-50 \%$ of HCCs. mTOR is involved in the regulation of cell metabolism and growth ${ }^{[23]}$. Therefore, various studies have suggested that MTOR inhibitors may have antineoplastic properties in HCC patients and mTOR inhibitors should be used after LT. In a meta-analysis of 2950 patients from five studies, sirolimus-based immunosuppression reduced the rate of tumor recurrence and improved overall survival $^{[24]}$. 
HCC recurrence following LT is an unfortunate event and associated with poor outcomes. In a recent meta-analysis, the median overall survival was 13 months following the diagnosis of HCC recurrence post$\mathrm{LT}^{[8]}$. Herein, supportive care was associated with the lowest survival rate of 3.3 months ${ }^{[8]}$. There is no standardized protocol regarding the type and frequency of post-LT cross-sectional imaging in surveillance of HCC LT recipients. It is important to note that more than $50 \%$ of patients develop tumor recurrences that are outside of liver (extrahepatic), therefore imaging limited to the liver may not be sufficient for the diagnosis of majority of HCC recurrences. We also note that AFP is a useful marker in post-LT HCC surveillance only for high-AFP-secreting tumors. Four patients in our study had pre-AFP levels of $>1000$ $\mathrm{ng} / \mathrm{mL}$. It is well known that patients with high AFP producing tumors have worse tumor biology and have worse outcomes ${ }^{[12,25]}$. HCC candidates need to have AFP of $\leq 1000 \mathrm{ng} / \mathrm{mL}$ to receive extra points to shorten the waiting period for liver transplantation ${ }^{[25]}$. The overall prognosis of HCC recurrence following LT is poor in the majority of cases and there are no available studies evaluating cost-effectiveness of surveillance protocols specific to this group of patients.

In conclusion, HCC recurrence post liver transplant is an unfortunate event associated with extremely poor survival. The majority of the cases are early recurrence occurring 1-2 years following liver transplantation. More than 50\% of HCC recurrences are extrahepatic. Therefore, post-liver transplant imaging confined to the liver may not be enough to detect all of the recurrences. In patients with AFP producing tumors, this marker may also be helpful to diagnose the HCC recurrence. There is no general consensus on the treatment for post liver transplant hepatocellular carcinoma recurrence. The current reports are mainly based on single-center retrospective experience.

\section{DECLARATIONS}

\section{Authors' contributions}

Acquisition of data, analysis and interpretation of data, drafting of the manuscript: Simsek C

Interpretation of the data, Final Editing and Critical Review of the manuscript for important intellectual content: Kim A

Acquisition of data, analysis and interpretation of data, drafting of the manuscript: Ma M

Review of the data, drafting of the manuscript and editing: Danis $\mathrm{N}$

Analysis and interpretation of data, statistical analysis: Gurakar M

Study concept and design, interpretation of data, critical revision manuscript for important intellectual content: Cameron AM

Study concept and design, interpretation of data, critical revision of the manuscript for important intellectual content: Philosophe B

Study concept and design, interpretation of data, statistical analysis, critical revision of the manuscript for important intellectual content: Garonzik-Wang J

Study concept and design, critical revision of the manuscript for important intellectual content: Ottmann S Study concept and design, acquisition of data, drafting of the manuscript, analysis and interpretation of data, revision of the manuscript for important intellectual content study supervision: Gurakar A

Study concept and design, acquisition of data, drafting of the manuscript, analysis and interpretation of data, critical revision of the manuscript for important intellectual content, study supervision: Saberi B

\section{Availability of data and materials}

Data source has been the Electronic Medical System. Please contact the corresponding author for unidentified data availability.

\section{Financial support and sponsorship}

This Research is partially supported by NIH Grants: R44 CA165312 - Development of a urine test for the early detection of liver cancer. U01 CA230690 - Pathway Specific Functional Biomarkers for the Early Detection of Liver Cancer. 


\section{Conflicts of interest}

All authors declared that there are no conflicts of interest.

\section{Ethical approval and consent to participate}

This retrospective study was approved by Johns Hopkins IRB NA_00028034.

\section{Consent for publication}

Not applicable.

\section{Copyright}

(c) The Author(s) 2020.

\section{REFERENCES}

1. Mazzaferro V, Regalia E, Doci R, Andreola S, Pulvirenti A, et al. Liver transplantation for the treatment of small hepatocellular carcinomas in patients with cirrhosis. N Engl J Med 1996;334:693-9.

2. Yao FY, Ferrell L, Bass NM, Watson JJ, Bacchetti P, et al. Liver transplantation for hepatocellular carcinoma: expansion of the tumor size limits does not adversely impact survival. Hepatology 2001;33:1394-403.

3. Mazzaferro V, Llovet JM, Miceli R, Bhoori S, Schiavo M, et al. Predicting survival after liver transplantation in patients with hepatocellular carcinoma beyond the Milan criteria: a retrospective, exploratory analysis. Lancet Oncol 2009;10:35-43.

4. Andreou A, Gul S, Pascher A, Schoning W, Al-Abadi H, et al. Patient and tumour biology predict survival beyond the Milan criteria in liver transplantation for hepatocellular carcinoma. HPB (Oxford) 2015;17:168-75.

5. Marshall AE, Rushbrook SM, Vowler SL, Palmer CR, Davies RJ, et al. Tumor recurrence following liver transplantation for hepatocellular carcinoma: role of tumor proliferation status. Liver Transpl 2010;16:279-88.

6. Llovet JM, Paradis V, Kudo M, Zucman-Rossi J. Tissue biomarkers as predictors of outcome and selection of transplant candidates with hepatocellular carcinoma. Liver Transpl 2011;17 Suppl 2:S67-71.

7. Fernandez-Sevilla E, Allard MA, Selten J, Golse N, Vibert E, et al. Recurrence of hepatocellular carcinoma after liver transplantation: is there a place for resection? Liver Transpl 2017;23:440-7.

8. de'Angelis N, Landi F, Carra MC, Azoulay D. Managements of recurrent hepatocellular carcinoma after liver transplantation: a systematic review. World J Gastroenterol 2015;21:11185-98.

9. Chok K. Management of recurrent hepatocellular carcinoma after liver transplant. World J Hepatol 2015;7:1142-8.

10. Kornberg A, Kupper B, Tannapfel A, Katenkamp K, Thrum K, et al. Long-term survival after recurrent hepatocellular carcinoma in liver transplant patients: clinical patterns and outcome variables. Eur J Surg Oncol 2010;36:275-80.

11. Roayaie S, Schwartz JD, Sung MW, Emre SH, Miller CM, et al. Recurrence of hepatocellular carcinoma after liver transplant: patterns and prognosis. Liver Transpl 2004;10:534-40.

12. Gurakar A, Ma M, Garonzik-Wang J, Kim A, Anders RA, et al. Clinicopathological distinction of low-AFP-secreting vs. high-AFPsecreting hepatocellular carcinomas. Ann Hepatol 2018;17:1052-66.

13. Guerrero-Misas M, Rodriguez-Peralvarez M, De la Mata M. Strategies to improve outcome of patients with hepatocellular carcinoma receiving a liver transplantation. World J Hepatol 2015;7:649-61.

14. Chok KS, Chan SC, Cheung TT, Chan AC, Fan ST, et al. Late recurrence of hepatocellular carcinoma after liver transplantation. World J Surg 2011;35:2058-62.

15. Zhang JA, Kwee SA, Wong LL. Late recurrence of hepatocellular carcinoma after liver transplantation. Hepatoma Res 2017;3:58-66.

16. Rubin J, Ayoub N, Kaldas F, Saab S. Management of recurrent hepatocellular carcinoma in liver transplant recipients: a systematic review. Exp Clin Transplant 2012;10:531-43.

17. Tabrizian P, Roayaie S, Schwartz ME. Current management of hepatocellular carcinoma. World J Gastroenterol 2014;20:10223-37.

18. Llovet JM, Ricci S, Mazzaferro V, Hilgard P, Gane E, et al. Sorafenib in advanced hepatocellular carcinoma. N Engl J Med 2008;359:378-90.

19. Cheng AL, Kang YK, Chen Z, Tsao CJ, Qin S, et al. Efficacy and safety of sorafenib in patients in the Asia-Pacific region with advanced hepatocellular carcinoma: a phase III randomised, double-blind, placebo-controlled trial. Lancet Oncol 2009;10:25-34.

20. Llovet JM, Montal R, Sia D, Finn RS. Molecular therapies and precision medicine for hepatocellular carcinoma. Nat Rev Clin Oncol 2018;15:599-616.

21. El-Khoueiry AB, Sangro B, Yau T, Crocenzi TS, Kudo M, et al. Nivolumab in patients with advanced hepatocellular carcinoma (CheckMate 040): an open-label, non-comparative, phase 1/2 dose escalation and expansion trial. Lancet 2017;389:2492-502.

22. Munker S, De Toni EN. Use of checkpoint inhibitors in liver transplant recipients. United European Gastroenterol J 2018;6:970-3.

23. Matter MS, Decaens T, Andersen JB, Thorgeirsson SS. Targeting the mTOR pathway in hepatocellular carcinoma: current state and future trends. J Hepatol 2014;60:855-65.

24. Liang W, Wang D, Ling X, Kao AA, Kong Y, et al. Sirolimus-based immunosuppression in liver transplantation for hepatocellular carcinoma: a meta-analysis. Liver Transpl 2012;18:62-9.

25. Mehta N, Dodge JL, Roberts JP, Hirose R, Yao FY. Alpha-fetoprotein decrease from $>1,000$ to $<500 \mathrm{ng} / \mathrm{mL}$ in patients with hepatocellular carcinoma leads to improved posttransplant outcomes. Hepatology 2019;69:1193-205. 